\title{
Multi-wavelength scanning Raman lidar simulations for the Cherenkov Telescope Array observatory
}

\section{Simulación del lidar Raman multiángulo para el observatorio CTA - Cherenkov Telescope Array}

\author{
Juan V. Pallotta(1, $\left.{ }^{*}\right)$ Pablo R. Ristori(1), Lidia A. Otero(1), \\ Alberto Etchegoyen(2), Eduardo Quel(1) \\ 1. UNIDEF-CEILAP-(CITEDEF-CONICET), UMI-IFAECI-CNRS (3351) - Buenos Aires, Argentina. \\ 2. ITeDA (CNEA CONICET - UNSAM) - Buenos Aires, Argentina. \\ ${ }^{(*)}$ Email: juanpallotta@gmail.com \\ Received / Recibido: 15/02/2014. Revised / Revisado: 05/05/2014. Accepted / Aceptado: 06/05/2014 \\ DOI: http://dx.doi.org/10.7149/OPA.47.2.91
}

\begin{abstract}
:
This paper discusses the multi-wavelength scanning Raman lidar being built at Lidar Division, CEILAP (CITEDEF-CONICET) in the frame of the Argentinean CTA (Cherenkov Telescope Array) collaboration. CTA is an initiative to build the next generation of ground-based instruments to collect very high energy gamma-ray radiation (greater than a few tens of $\mathrm{GeV}$ ). The atmospheric conditions are of major interest to CTA, and lidars are requested to acquire atmospheric profiles fast, accurately and in the line of sight of the event. Due to the expected low aerosol optical depth of the future site, the short observation period as well as the extension of the observation, an enhanced collection area is required. Based on this constraints, and energy laser pulse, a backscatter lidar signal simulation was performed to estimate the main characteristics of the system. To derive these features, a SNR (signal-to-noise ratio) was also simulated to derive the total number of mirrors to achieve a good quality signal over the whole tropopause. Raman capabilities were added in the UV and VIS wavelengths to retrieve the spectral characteristics of the aerosol extinction and the water vapor profile. The results of the simulation, and the main characteristics derived are shown.
\end{abstract}

Key words: Lidar, Aerosols, Atmosphere, CTA Observatory.

\section{RESUMEN:}

En este trabajo se describe la simulación del lidar Raman multiángulo que se está desarrollando en la división lidar del CEILAP (CITEDEF-CONICET), en el marco de la colaboración argentina del observatorio CTA (Cherenkov Telescope Array). Este Observatorio es una iniciativa para construir una nueva generación de instrumentos para detectar radiación gamma de muy altas energías (superior a $10 \mathrm{GeV}$ ). En base a restricciones de construcción y de parámetros atmosféricos, se realizó la simulación de la relación S/R (señal/ruido) para obtener el área total de colección del sistema lidar. Este sistema está planeado para medir señales Raman de nitrógeno y vapor de agua provenientes de las líneas de $355 \mathrm{~nm}$ y $532 \mathrm{~nm}$.

Palabras clave: Lidar, Aerosoles, Atmósfera, Observatorio CTA.

\section{REFERENCES AND LINKS / REFERENCIAS Y ENLACES}

[1]. http://www.cta-observatory.org/

[2]. M. Doro, M. Gaug, O. Blanch, L. Font, D. Garrido, A. Lopez-Oramas, M. Martinez, "Towards a full Atmospheric Calibration system for the Cherenkov Telescope Array", Proceedings of the International Cosmic Ray Conference, Rio de Janeiro, Brazil (2013). http://arxiv.org/abs/1307.3406

[3]. A. Lopez Oramas, O. Abril, O. Blanch Bigas, J. Boix, V. Da Deppo, M. Doro, L. Font, D. Garrido, M. Gaug, M.Martínez, G. Vasileiadis, "The IFAE/UAB and LUPM Raman LIDARs for the CTA Observatory", 
Proceedings of the International Cosmic Ray Conference, Rio de Janeiro, Brazil (2013). http://arxiv.org/abs/1307.5092v1

[4]. M. Tesfaye, V. Sivakumar, J. Botai, G. Mengistu Tsidu, "Aerosol climatology over South Africa based on 10 years of Multiangle Imaging Spectroradiometer (MISR) data (2011)", J. Geophys. Res. 116 (D20), 2156-2202, (2011). DOI

[5]. J. Ackerman. "The extinction-to-backscatter ratio of tropospheric aerosol: A numerical study", J. Atmos. Oceanic Technol. 15, 1043-1050 (1998). DOI

[6]. "Photon counting using photomultiplier tubes". Technical information of Hamamatsu Company. http://www.hamamatsu.com/jp/en/technology/innovation/photoncounting/index.html (Visited May 2014)

[7]. J. V. Pallotta, P. Pablo, L. Otero, F. Chouza, R. D’elia, F. González, A. Etchegoyen, E. Quel, "Remote control and telescope auto-alignment system for multiangle LIDAR under development at CEILAP, Argentina", Proceedings of AtmoHead 2013: Atmospheric Monitoring for High Energy Astroparticle Detectors, (2013). arXiv:1309.6535.

\section{Introduction}

The Cherenkov Telescope Array Consortium (CTA) [1] contemplates the design, construction and the operation of two observatories to detect galactic and extragalactic gamma ray sources at energies ranging between a few of $10^{10} \mathrm{eV}$ and $10^{14} \mathrm{eV}$. These observatories will be deployed at each hemisphere for full sky map coverage. Each Observatory will consist of a telescope array sensitive to the atmospheric-generated Cherenkov radiation that will improve the performance of the present-day Cherenkov telescope observatories. The goals proposed for CTA will be attained using an array of multiple telescopes distributed over an area between 1 and $10 \mathrm{~km}^{2}$ in the northern and southern hemispheres, respectively located.

These sites are expected to have outstanding optical and atmospheric conditions at heights ranging from 1500 to $3800 \mathrm{~m}$ above the sea level. Real time aerosol extinction profile observations over the observatories are important to determine the collected Cherenkov light attenuation. This reduces the number of discarded runs by enhancing the quality of the spectral analysis. In this sense multiangle Raman lidars play a major role as they are capable to monitor optical properties of clouds and aerosols in the line of sight of the measurements.

As the atmospheric extinction will be retrieved by inverting Raman signals [2,3], it is critical to improve their signal to noise ratio (SNR) by increasing the laser pulse energy, repetition rate and the collection area. Ultraviolet wavelengths are preferred to visible ones due to of the magnitude increase of the Raman effect and Cherenkov spectral properties as well as the quantum efficiency of the detectors at those wavelengths. All the lidars proposed are intended to measure the atmospheric nitrogen Raman return of the third harmonic of a Nd:YAG laser emission to retrieve the most significant Raman profile while the one obtained from the second harmonic will help to determine the aerosol spectral extinction properties.

\section{Hardware constraints for the multiangle lidar construction}

Hardware features define a set of constraints in the simulation, and have a great influence in the lidar signal. Next, a list of the most important key factors for the design:

\subsection{Laser}

A Continuum Inlite II-50 laser is being used as an emission system. This is a flash lamp pumped, Qswitched, Nd:YAG solid state laser that emits pulses of $60 \mathrm{~mJ}$ (at $532 \mathrm{~nm}$ ) with a maximum repetition rate of $50 \mathrm{~Hz}$. The laser is designed for operation under harsh environmental conditions like industrial environments, to reduce misalignment due to changes in temperature or vibration.

\subsection{Reception telescopes}

Multiple of single light glass, $400 \mathrm{~mm}$ diameter and $1 \mathrm{~m}$ focal length mirrors compose the collection surface. This solution was preferred to a single, short focal length mirror to avoid dealing with higher angular-dependent 
deformation and aberrations at the focal plane. It also reduces the overall cost and permits a fast and relatively simple partial replacement of the collection structure when required. The number of mirrors was estimated to perform slant path aerosol detection over the whole troposphere with a reasonable SNR.

\subsection{Acquisition time:}

We focus this design to obtain Raman lidar profiles fast and accurate, taking the minimum acquisition time. We use as reference the nitrogen Raman line from $355 \mathrm{~nm}(387 \mathrm{~nm})$ while ensuring good levels in this line, we will get better levels in the others.

\section{Lidar signal simulation}

To run the simulation, hardware and atmospheric parameters were modelled in order to compute the expected lidar signals.

\subsection{Hardware model}

Hardware parameters used in the simulation are listed in Table I. We define the background value as the voltage measured at the photomultiplier's anode using a $50 \mathrm{ohm}$ load. This value is being overestimated as these measurements will be done in moonless nights.

\subsection{Atmospheric model}

The parameters for the atmospheric aerosol optical properties were modelled based on Aerosol Robotic NETwork (AERONET) and Multi-angle Imaging Spectro-Radiometer (MISR) aerosol data at the CTA candidate sites. These instruments are owned by NASA, and are well correlated [4]. The LR's values were obtained from [5], for desert aerosols. These values are summarized in Table II.

TABLE I

Hardware parameters for the lidar simulation.

\begin{tabular}{|c|c|c|c|}
\hline & \multicolumn{2}{|c|}{ Parameter } & Value \\
\hline \multirow{4}{*}{ Emission } & \multicolumn{2}{|c|}{ Wavelengths } & $355,532 \mathrm{~nm}$ \\
\hline & \multicolumn{2}{|c|}{ Energy pulse @ 355, 532 nm } & $20,60 \mathrm{~mJ}$ \\
\hline & \multicolumn{2}{|c|}{ Repetition rate } & $50 \mathrm{~Hz}$ \\
\hline & \multicolumn{2}{|c|}{ Overall transmission optics efficiency } & 0.8 \\
\hline \multirow{7}{*}{ Detection } & \multicolumn{2}{|c|}{ Single mirror diameter } & $40 \mathrm{~cm}$ \\
\hline & \multicolumn{2}{|c|}{ Telescope $f \#$} & 2.5 \\
\hline & \multicolumn{2}{|c|}{ Reception optics efficiency } & 0.2 \\
\hline & \multirow{2}{*}{ Background Signal } & $355 \mathrm{~nm}$ & \multirow{2}{*}{$5 \mathrm{uV}$} \\
\hline & & $532 \mathrm{~nm}$ & \\
\hline & \multicolumn{2}{|c|}{ Bin Size Analog } & $7,5 \mathrm{~m}$ \\
\hline & \multicolumn{2}{|c|}{ Max Count Rate (Raman) } & $250 \mathrm{Mhz}$ \\
\hline
\end{tabular}


TABLE II

Atmospherics parameters for simulation:

\begin{tabular}{|c|c|}
\hline \hline Parameter & Value \\
\hline \hline AOD @ $532 \mathrm{~nm}$ & $0.3976\left(^{*}\right)$ \\
\hline AOD @ $355 \mathrm{~nm}$ & $0.6727\left(^{*}\right)$ \\
\hline ABL heigth & $2000 \mathrm{~m}$ \\
\hline LR @ 532 nm & $19.93 \mathrm{sr}$ \\
\hline LR @ $355 \mathrm{~nm}$ & $41.91 \mathrm{sr}$ \\
\hline \hline
\end{tabular}

${ }^{*}$ ) Based on a reference AOD of $0.2 @ 446 \mathrm{~nm}$, and Angstrom coefficient of 1.3 .

\subsection{Simulation}

With hardware and atmospheric model summarized in sub-section 3.1 and 3.2, we are able to compute the lidar signal simulation for elastic and nitrogen Raman lines. Next figures shows the simulations for elastic backscattered and the nitrogen Raman returns, from a laser emission of 355 and $532 \mathrm{~nm}$.

The next job is to compute the SNR focused on the $355 \mathrm{~nm}$ nitrogen Raman line (387 nm). Regardless of Raman water vapour line, $387 \mathrm{~nm}$ is the weakness Raman line, and if we assured a good SNR for this one, will be better for the rest of the lines acquired.
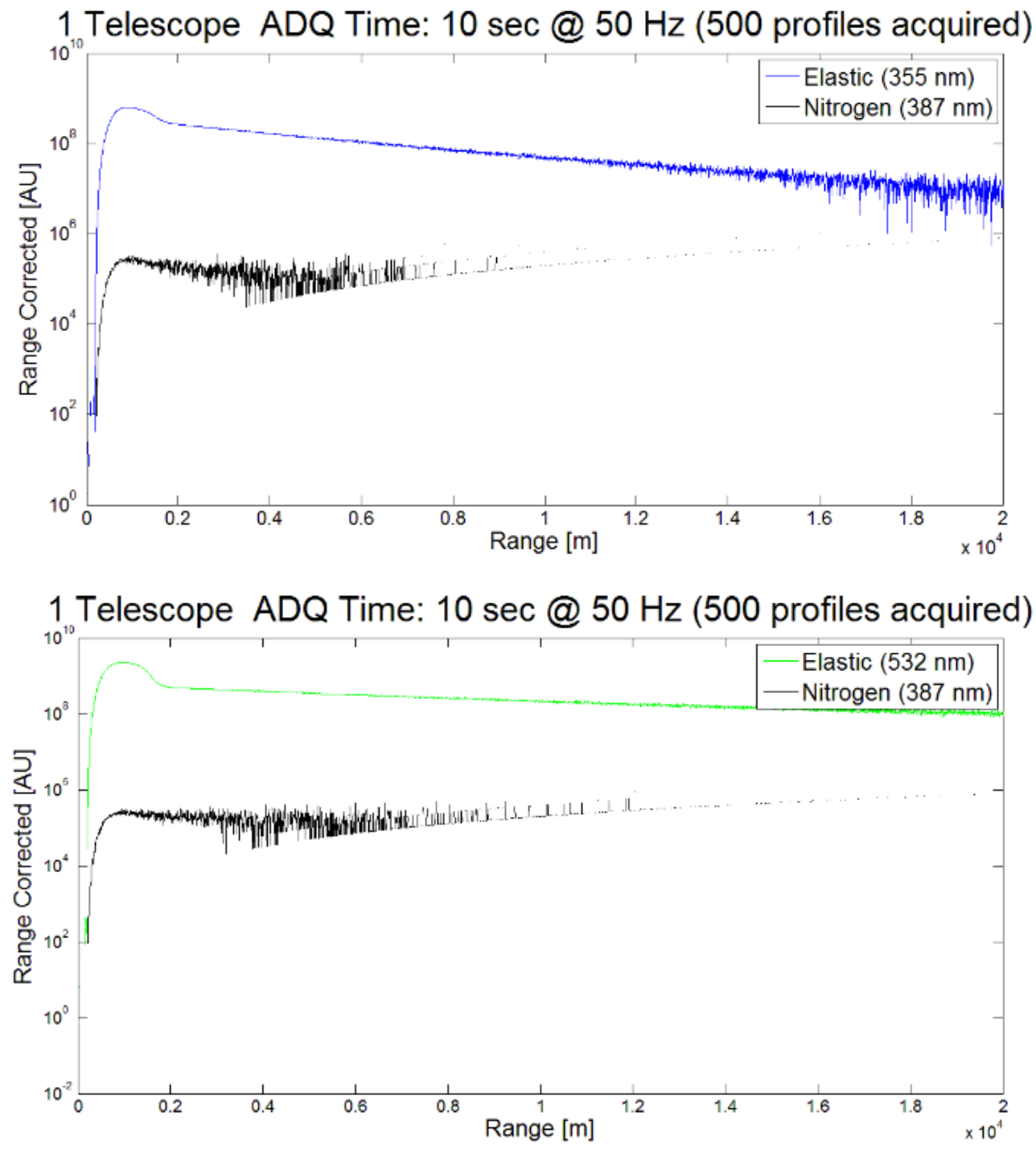

Fig. 1. Simulated lidar signal return for a single telescope and 10 seconds of integration time. 


\section{Signal-to-noise ratio simulation}

The aim of the simulation is to obtain the quantity of telescopes (defined in sub-section 2.2) required to reach a SNR higher than one over the troposphere. As stated before, these constraints were set to the $387 \mathrm{~nm}$ line, which is the nitrogen backscattered line of the $355 \mathrm{~nm}$. This quantity can be derived using the SNR equation from [6].

Let Nphot be the number of photons of the lidar signal acquired with a single mirror, NphotBKG its background noise, and Acc is the accumulated profiles, we can express the SNR as:

$$
\mathrm{SNR}=\frac{\text { Nphot }}{\sqrt{\frac{N p h o t+N p h o t B K G}{A c c}}} .
$$

Expliciting the number of telescopes (numTel), we can write:

$$
\begin{array}{r}
\text { SNR }=\frac{\text { numTel } \cdot \text { Nphot }}{\sqrt{\text { numTel }\left(\frac{\text { Nphot }+ \text { NphotBKG }}{A c c}\right)}}= \\
=\frac{\sqrt{\text { numTel }} \cdot \text { Nphot }}{\sqrt{\frac{\text { Nphot }+ \text { NphotBKG }}{A c c}}} .
\end{array}
$$

And the numbers of telescope for $\mathrm{SNR}=1$ is:

$$
\text { numTel }=\frac{\text { Nphot }+N p h o t B K G}{A c c \cdot N p h o t^{2}} .
$$

With this last equation, we can obtain the minimum quantity of telescopes to assure a $\mathrm{SNR}=1$ which is inversely proportional to the acquisition time or the number of accumulated profiles (Acc). Plotting Eq. (3) for $387 \mathrm{~nm}$ simulated lidar signal, and for a set of different acquisition time, a useful information can be extracted.

Based on last plot, we can define an acquisition time of 10 seconds (500 profiles at $50 \mathrm{~Hz}$ ), and 6 telescopes to reach a SNR>1 over the whole troposphere for $387 \mathrm{~nm}$. This assures a better SNR for the rest of the lines acquired, as can be seen in Fig. 3 .

We can conclude that 6 telescopes and $10 \mathrm{sec}$ of acquisition time will be more than enough to assure a good lidar signal quality to fit the CTA constraints.

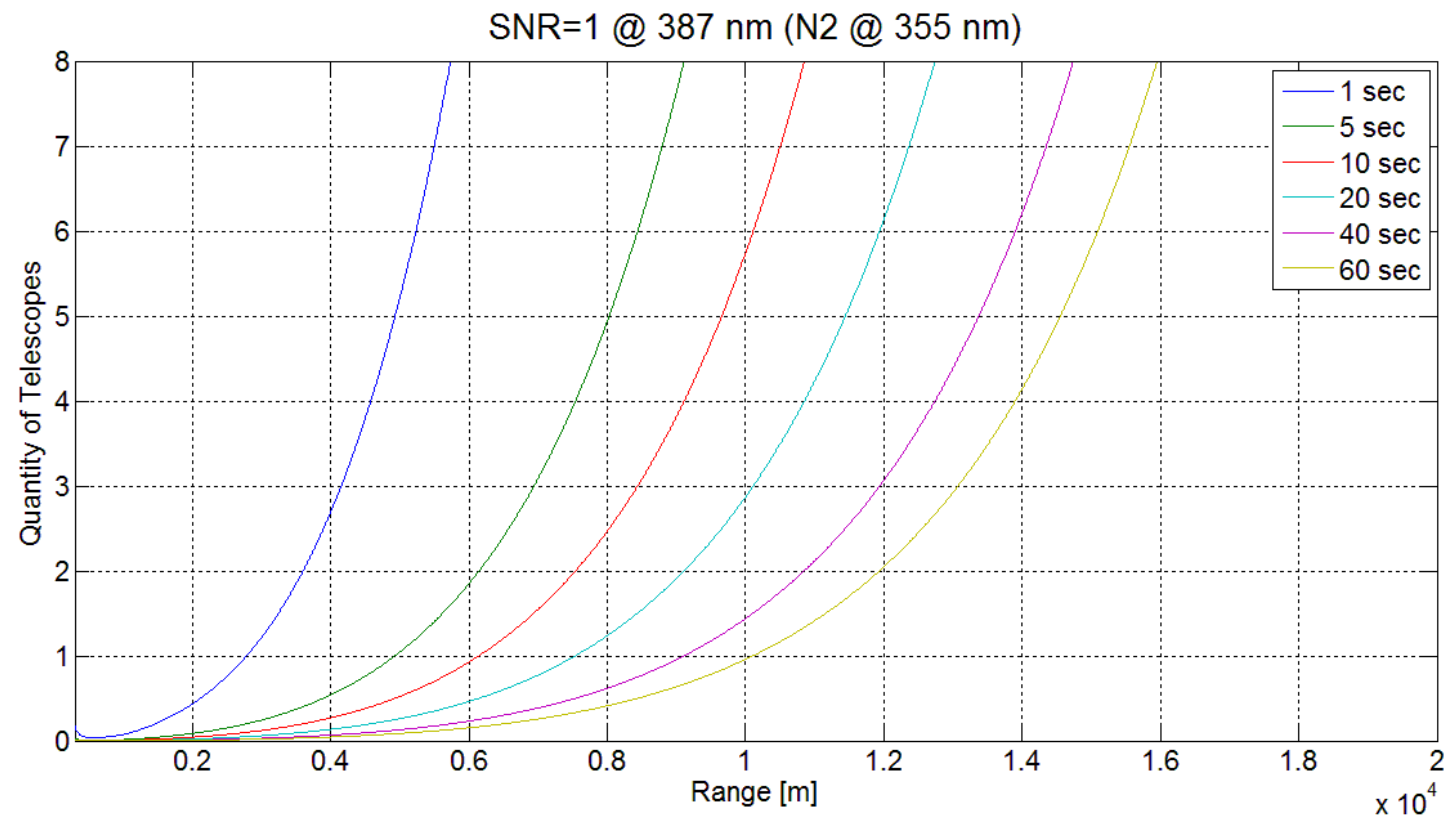

Fig. 2. Quantity of telescopes needed as a function of range and acquisition time 


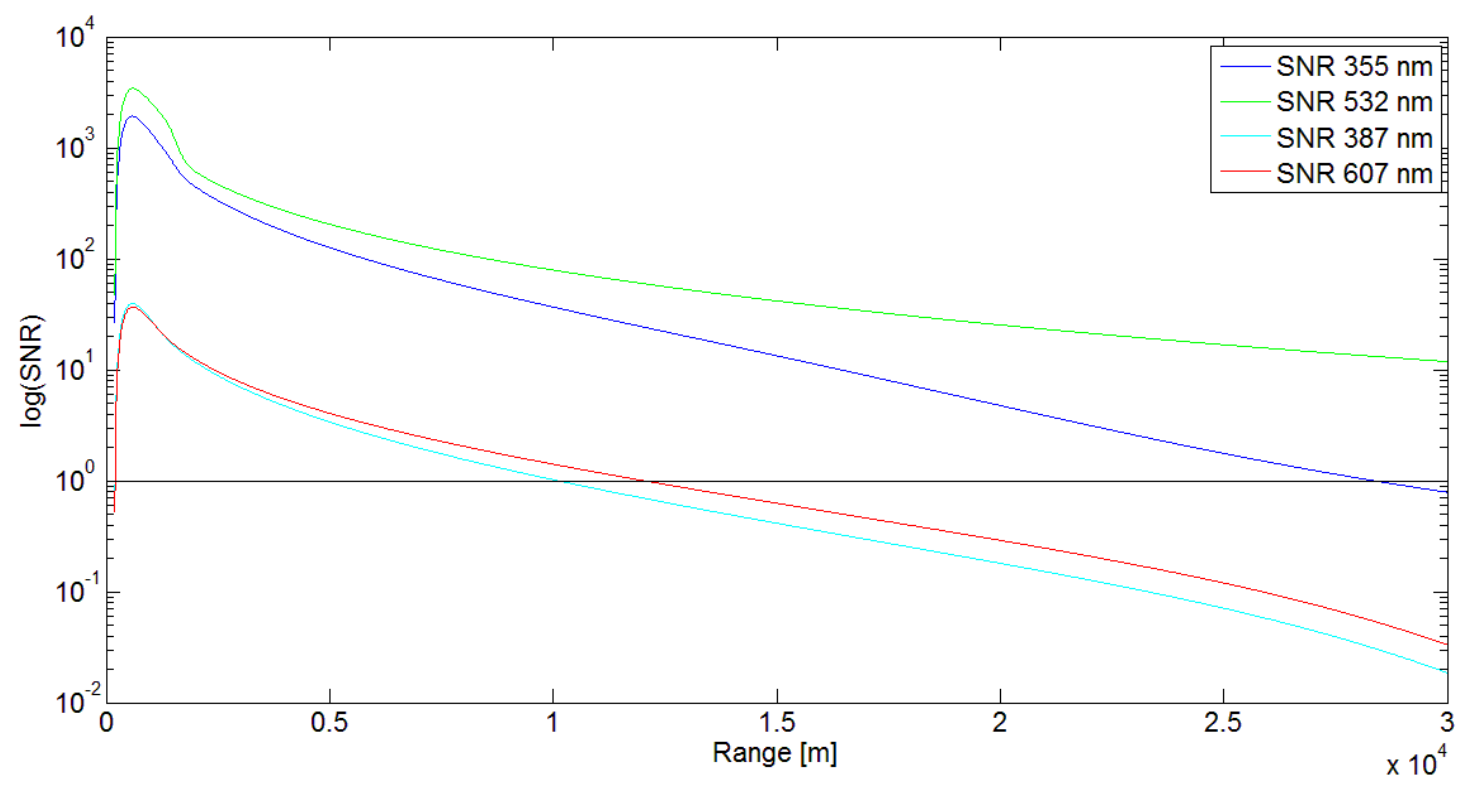

Fig. 3. SNR simulation for elastics lines (355 and $532 \mathrm{~nm}$ ) and its nitrogen's Ramans lines.

\section{Mirrors scanning system}

In order to hold the six mirrors with scanning capabilities, a scanning bench is under development and planned to be finished at the end of this year. This work is being done with the Mechanical Department of CITEDEF (Fig. $4(\mathrm{a}))$.

The main structure holds a hexagonal honeycomb (Fig. 4(b)) where is mounted all mirrors holders and laser. Each of the six mirrors is mounted on a steerable frame, equipped with two stepper motors for tilting over two orthogonal axes. This is part of telescope auto-alignment system under development [7], and aims to keep good levels of signals over all ranges. The tilt angle of the telescopes is driven by a set of stepper motors, handled by a microcontroller, and tends to minimize the signal loss due to misalignments.

\section{Conclusions}

The presented multiwavelength scanning Raman lidar will be able to provide, in a short period of time, spectrally-resolved aerosol extinction profiles to characterize the atmospheric transmission at any required line of sight. This
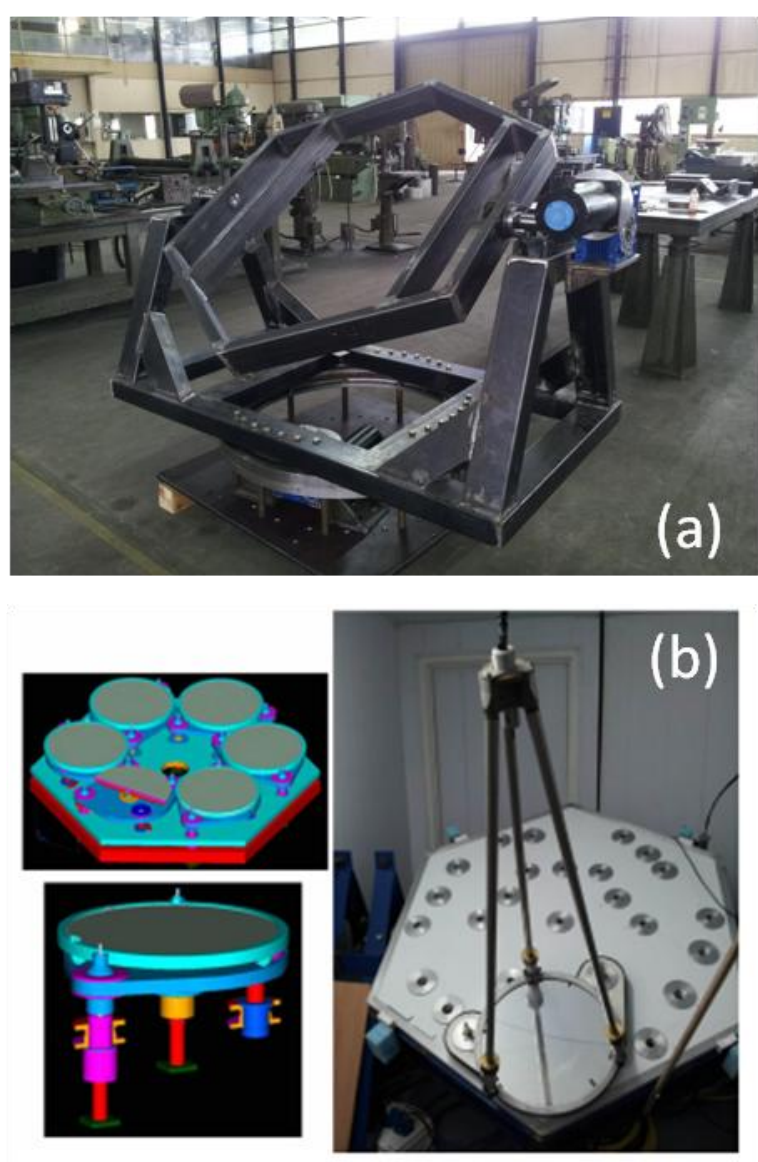

Fig. 4. (a) Scanning bench under development. (b) Mirror holder under test mounted on hexagonal honey-comb plate. 
work analyses the performance of multiangle Raman lidar design based on several instrumentation and atmospheric constraints. This assures that the performance of the system will accomplish the functionality requirements of the CTA Observatory. From our results we conclude that this new design will be able to acquire backscatter returns with an adequate signal to noise ratio for most of the scans conditions in the whole troposphere as well as in the tropopause - low stratosphere if needed.

The modularity of the telescope system will permit the system maintenance and optimization while being operated reducing non- operational times. The collaboration of CEILAP, IFAE/UAB and LUPM to improve their lidar systems will permit to attain the requested goals in terms of system construction, lidar testing, instrumentation control and lidar signal processing.

\section{Acknowledgements}

Authors wish to thank CITEDEF main workshops technicians, Mario Proyetti, Fabian Jeric and Jos Luis Luque from the CEILAP workshop for their support on this development. 